\title{
Nature of the Epidemic Threshold for the Susceptible-Infected-Susceptible Dynamics in Networks
}

\author{
Marian Boguñá, ${ }^{1}$ Claudio Castellano, ${ }^{2,3}$ and Romualdo Pastor-Satorras ${ }^{4}$ \\ ${ }^{1}$ Departament de Física Fonamental, Universitat de Barcelona, Martí i Franquès 1, 08028 Barcelona, Spain \\ ${ }^{2}$ Istituto dei Sistemi Complessi (ISC-CNR), via dei Taurini 19, I-00185 Roma, Italy \\ ${ }^{3}$ Dipartimento di Fisica, "Sapienza" Università di Roma, Piazzale Aldo Moro 2, I-00185 Roma, Italy \\ ${ }^{4}$ Departament de Física i Enginyeria Nuclear, Universitat Politècnica de Catalunya, Campus Nord B4, 08034 Barcelona, Spain
}

(Received 21 May 2013; published 7 August 2013)

\begin{abstract}
We develop an analytical approach to the susceptible-infected-susceptible epidemic model that allows us to unravel the true origin of the absence of an epidemic threshold in heterogeneous networks. We find that a delicate balance between the number of high degree nodes in the network and the topological distance between them dictates the existence or absence of such a threshold. In particular, small-world random networks with a degree distribution decaying slower than an exponential have a vanishing epidemic threshold in the thermodynamic limit.
\end{abstract}

DOI: 10.1103/PhysRevLett.111.068701

PACS numbers: $89.75 . \mathrm{Hc}, 05.40 . \mathrm{Fb}$

The accurate theoretical understanding of epidemic thresholds on complex networks is a pressing challenge in the field of network science [1-3]. Indeed, such knowledge has potential practical applications in the design of optimal immunization programs [4,5] and may shed light on the behavior of the viral spreading of rumors, fads, and beliefs [6,7]. In this respect, large research effort has recently been devoted to the study of the susceptibleinfected-susceptible (SIS) epidemic model [8], the simplest model of epidemic spreading showing an absorbing state phase transition between a healthy and an endemic phase at a critical value of the effective infective rate $\lambda$ [9]. The behavior of the SIS model is particularly relevant in the case of highly heterogeneous networks, for which a vanishing epidemic threshold in the thermodynamic limit has been pointed out [10]. Recently, a scientific controversy has arisen concerning the location and the real nature of the epidemic threshold in this kind of network [11-14]. In this Letter, we provide strong analytical and numerical arguments showing that the threshold asymptotically vanishes in any network with a degree distribution decaying slower than exponentially, thus clarifying the physical origin of this behavior.

In the SIS model, individuals can be in one of two states, either susceptible or infected. Susceptible individuals become infected by contact with infected individuals at rate $\lambda$ times the number of infected contacts. Infected individuals, on the other hand, become spontaneously healthy again at rate $\mu$ that, without loss of generality, is set to unity. The original approach to the dynamics of the SIS model [10] was based on the so-called heterogeneous mean-field (HMF) theory [15,16], which neglects both dynamical and topological correlations. To do so, the actual quenched structure of the network-given by its adjacency matrix $A_{i j}$ [3] -is replaced by an annealed version, in which edges are constantly rewired at a rate much faster than that of the epidemics, while preserving the degree distribution $P(k)$. According to HMF theory, the epidemic threshold of the SIS model takes the form $\lambda_{c}^{\mathrm{HMF}}=\langle k\rangle /\left\langle k^{2}\right\rangle[10]$, where $\langle k\rangle$ and $\left\langle k^{2}\right\rangle$ are the first and second moments of $P(k)$ [3]. Many real networks have a heterogeneous degree distribution, often scaling as a power law (PL), $P(k) \sim k^{-\gamma}$ [1-3]. This implies that the second moment diverges with the maximum degree $k_{\max }$ for a degree exponent $\gamma<3$, leading to a threshold scaling $\lambda_{c}^{\mathrm{HMF}} \sim k_{\max }^{\gamma-3}$, which vanishes in the thermodynamic limit. On the other hand, for $\gamma>3$, the second moment is finite and consequently so is the epidemic threshold.

While HMF theory represents an exact result in the case of annealed networks [17,18], its validity for real (quenched) networks is limited. Indeed, an important improvement over HMF theory is given by the quenched mean-field theory (QMF) [19-21] which, while still neglecting dynamical correlations, takes into account the full form of $A_{i j}$. Within this framework, the epidemic threshold is predicted to be $\lambda_{c}^{\mathrm{QMF}}=1 / \Lambda_{N}$, where $\Lambda_{N}$ is the largest eigenvalue of the adjacency matrix. Given the scaling of $\Lambda_{N}$ with the maximum degree, $\Lambda_{N} \sim$ $\max \left\{\sqrt{k_{\max }},\left\langle k^{2}\right\rangle /\langle k\rangle\right\}$ [22], QMF theory predicts the same result as HMF theory for $\gamma<5 / 2$, while for $\gamma>5 / 2$ it leads to $\lambda_{c}^{\mathrm{QMF}} \sim 1 / \sqrt{k_{\max }}$, that is, to a vanishing threshold for any value of $\gamma$ (even for $\gamma>3$ ) in the thermodynamic limit [11]. The prediction of QMF theory has been validated for $\gamma<3$ by means of large-scale numerical simulations based on the quasistationary state method [18]. Numerical evidence for $\gamma>3$ is, however, less convincing and has led to the following two criticisms.

Goltsev et al. [13] have considered, within the QMF framework, the effects of eigenvector localization on the steady state of the SIS model. According to their observations, in PL networks with $\gamma<5 / 2$, the principal eigenvector is delocalized, which implies that the density of 
infected nodes is finite above $\lambda_{c}^{\mathrm{QMF}}$. However, for $\gamma>5 / 2$, the principal eigenvector is localized, meaning that above $\lambda_{c}^{\mathrm{QMF}}$ the system is active but activity is concentrated around the hubs and their neighbors, leading to a number of infected nodes that scales sublinearly with system size and, therefore, does not constitute a true endemic state. The endemic state should, instead, appear at a different threshold, inversely proportional to the eigenvalue of the upper delocalized state, and approximately corresponding to the HMF value [13]. Therefore, the true threshold to the endemic state would have a finite value for $\gamma>3$, at odds with the interpretation of QMF theory made in [11].

This view is further pursued by Lee et al. [14] by partly taking into account dynamical correlations. Their argument is as follows. Slightly above the QMF threshold, hubs in a PL network become active but their activity is restricted to their immediate neighborhood. This activity has a characteristic lifetime $\tau(k, \lambda)$ depending on the degree and the value of the spreading rate $\lambda$. When hubs are directly connected to each other (the case of a clustered network, in the nomenclature of Ref. [14]), activity can be transferred between hubs if the lifetime $\tau(k, \lambda)$ is sufficiently large. In this case, above $\lambda_{c}^{\mathrm{QMF}}$ the network is able to support an endemic state, characterized by the mutual reinfection of connected hubs. In the case of unclustered networks, however, hubs are not directly connected and the reinfection mechanism does not work. Thus, the authors of Ref. [14] claim that the state above $\lambda_{c}^{\mathrm{QMF}}$ is just a Griffiths phase [23], where the density of infected nodes decays with time more slowly than exponentially (logarithmically indeed), while the actual epidemic threshold is located at a higher, finite value of $\lambda$. Within this picture a true zero epidemic threshold in the thermodynamic limit occurs only for $\gamma<3$.

While the arguments presented in Refs. [13,14] are appealing and, apparently, lead to the conclusion that the threshold is finite in random PL networks with $\gamma>3$, here we reconsider the problem and provide analytical and numerical evidence pointing in the opposite direction, namely, a vanishing epidemic threshold for any smallworld network with a degree distribution decaying slower than exponentially, in particular, power-law networks with any $\gamma$. To confirm this prediction, we propose a numerical approach, based on the scaling analysis of the survival time of the infection process, which is able to provide very accurate estimates of the epidemic threshold even in the regime where the quasistationary state method is unreliable.

Our analytical approach is based on the consideration of dynamical correlations, as in [14], but not restricted to direct neighbors. The argument of [14] assumes that a zero epidemic threshold can only occur in clustered networks, when hubs are directly connected to each other and can reinfect each other within a time smaller than the characteristic healing time $\tau$. However, as already pointed out in Ref. [24], a direct connection is not a necessary condition for the possibility of hub reinfection. Instead, we should properly consider the possibility of reinfection between two vertices $i$ and $j$, separated by a topological distance $d_{i j}$, possibly larger than 1 . Indeed, the epidemic threshold predicted by the HMF theory is actually based on the local properties of the network alone, assuming that the local structure will replicate in a treelike fashion forever, preserving only the statistical properties of the network. Then, above $\lambda_{c}^{\mathrm{HMF}}$, we expect that a perturbation originated in a node will be able to propagate as a supercritical branching process forever. Below this threshold, this process is not possible. Still, as we show below, the epidemic can sustain itself, due to perturbations which propagate up to distances of order $\ln (N)$, where $N$ is the network size.

To take into account dynamical correlations over distant neighbors, we replace the original SIS dynamics by a modified description of the SIS process valid over coarse-grained time scales. On such longer temporal intervals, it is possible that a given infected node $i$ propagates the infection to any other node $j$ in the network via a sequence of microscopic infection events of intermediate, nearest neighbors nodes. The infective rate $\lambda$ is then replaced by the effective rate $\bar{\lambda}\left(d_{i j}, \lambda\right)$ at which the infected node $i$ infects any other node $j$ in the network when the process is mediated by a chain of $d_{i j}-1$ intermediate nodes. On the coarse-grained time scale also the recovery rate $\delta$ of node $i$ is replaced by an effective rate $\bar{\delta}\left(k_{i}, \lambda\right)$. Overall, the evolution of the SIS dynamics over the coarsegrained time scale is then given by

$\frac{d \rho_{i}(t)}{d t}=-\bar{\delta}\left(k_{i}, \lambda\right) \rho_{i}(t)+\sum_{j \neq i} \bar{\lambda}\left(d_{i j}, \lambda\right) \rho_{j}(t)\left[1-\rho_{i}(t)\right]$,

which is defined on a fully connected graph. The parameters $\bar{\delta}$ and $\bar{\lambda}$ reflect in this description the structure of the original network. On long time scales node $i$ is considered as susceptible only when the node and all of its nearest neighbors in the original graph are susceptible; hence, its recovery rate is $\bar{\delta}\left(k_{i}, \lambda\right)=\tau^{-1}\left(k_{i}, \lambda\right) \approx e^{-a(\lambda) k_{i}}$ (see the Supplemental Material for numerical results and an analytical argument [25]), where $a(\lambda)$ is a smooth growing function of $\lambda$. To evaluate the effective infective rate it is convenient to assume that paths connecting nodes are independent and made of nodes of degree 2 . This leads to the expression $\bar{\lambda}\left(d_{i j}, \lambda\right) \approx \lambda e^{-b(\lambda)\left(d_{i j}-1\right)}$, with $b(\lambda)=$ $\ln (1+1 / \lambda)$ (see Supplemental Material for an analytical derivation and a numerical validation [25]). The assumptions made for determining $\bar{\lambda}$ are clearly not true in a real network because there are many paths connecting the same pair of nodes, and intermediate nodes have, in general, degrees larger than 2 . This implies that the infective rate between two nodes that we use in the coarse-grained SIS dynamics is smaller than the real one. Therefore, Eq. (1) will provide an upper bound for the true epidemic threshold of the original SIS dynamics and, thus, the absence of 
an epidemic threshold of the former will imply also its absence in the latter.

Equation (1) can be applied to any network. We can, however, get deeper insights in the case of small-world random graphs, in which the average internode topological distance takes the form [26]

$$
d_{k k^{\prime}}=1+\frac{\ln \left(\frac{N\langle k\rangle}{k k^{\prime}}\right)}{\ln \kappa},
$$

where $\kappa=\left\langle k^{2}\right\rangle /\langle k\rangle-1$ is the average branching factor of the network. Using the approximation Eq. (2) allows us to coarse grain Eq. (1) for degree classes. After defining $\rho_{k}(t) \equiv \sum_{\operatorname{deg}(i)=k} \rho_{i}(t) / N P(k)$ and plugging Eq. (2) into Eq. (1), we obtain

$$
\begin{aligned}
\frac{d \rho_{k}(t)}{d t}= & -\bar{\delta}(k, \lambda) \rho_{k}(t)+\lambda N\left[\frac{k}{N\langle k\rangle}\right]^{b(\lambda) / \ln \kappa} \\
& \times \sum_{k^{\prime}} k^{[b(\lambda) / \ln \kappa]} P\left(k^{\prime}\right) \rho_{k^{\prime}}(t)\left[1-\rho_{k}(t)\right] .
\end{aligned}
$$

Note that the use of Eq. (2) implies that the local propagation of the infection among directly connected nodes is neglected; only reinfections between distant $(\sim \ln N)$ nodes are taken into account. By performing a linear stability analysis of this equation, we can see that the critical epidemic threshold of the coarse-grained SIS dynamics $\lambda_{c}$ is the solution of the transcendental equation (see Supplemental Material for a detailed derivation [25])

$$
1=\lambda N \sum_{k=k_{\min }}^{k_{\max }} P(k) \tau(k, \lambda)\left[\frac{k^{2}}{N\langle k\rangle}\right]^{b(\lambda) / \ln \kappa} .
$$

In general, the maximum degree of the network $k_{\max }$ is a growing function of $N$. If we further assume that the degree distribution decays slower than an exponential, Eq. (4) can be approximated as the integral near the upper bound $k_{\max }$; i.e.,

$$
1=\frac{\lambda}{a(\lambda)} e^{a(\lambda) k_{\max }-[b(\lambda) / \ln \kappa] \ln \left[N\langle k\rangle / k_{\max }^{2}\right]-\ln \left[1 / N P\left(k_{\max }\right)\right]} .
$$

When $P(k)$ decays slower than an exponential, $k_{\max }$ grows faster than $\ln N$. Therefore, as the system size grows while keeping $\lambda$ fixed, there is a point where the first term in the exponential becomes larger than the other two (negative) terms and, eventually, the right-hand side of Eq. (4) becomes larger than 1 . As a consequence, the epidemic threshold of the coarse-grained SIS dynamics starts decreasing as $N$ increases, thus going to zero in the thermodynamic limit. Making the additional assumption that $a(\lambda) \approx a \lambda^{2}$ for $\lambda \ll 1$ (which is compatible with numerical simulations, see Supplemental Material [25]), we conclude that the upper bound of the epidemic threshold decreases as $1 / \sqrt{k_{\max }}$, with additional logarithmic corrections to scaling. Interestingly, this scaling is similar to the one predicted by the QMF theory. However, in our case, the threshold marks the onset of a true endemic state where a finite fraction of all nodes of the system are active.

The case of non-small-world networks can be considered along the same lines. Unfortunately, a general formula for the average topological distance as a function of the nodes' degrees is not known. Nevertheless, the absence of long-range connections in non-small-world networks suggests that node degree is not as determinant as in the case of small-world ones. Thus, to get some understanding, we assume an internode distance independent of the degree and scaling as a power law with system size, i.e., $d=1+$ $\alpha N^{\beta}$. An analysis similar to the case of small-world networks (see Supplemental Material [25]) concludes that non-small-world networks have a vanishing epidemic threshold only if $k_{\max }$ grows faster than $N^{\beta}$. This result explains the finite epidemic threshold in the $(3,3)$-flower model [27] found in Ref. [14], even if the model generates a PL network with $\gamma=1+\ln 6 / \ln 2 \approx 3.58$. Indeed, this model generates a non-small-world network with $\beta=\ln 3 / \ln 6$, whereas $k_{\max } \sim N^{\ln 2 / \ln 6}$ [27].

To check the accuracy of our theory, we propose a method to estimate the critical point of absorbing state phase transitions. The method is based on the analysis of individual realizations of the process starting with a single infected node. Each realization is characterized by its lifetime $T$ and coverage $C$, where the latter is defined as the fraction of distinct nodes ever infected during the realization. In the thermodynamic limit, realizations can be of two types: finite or endemic. Finite realizations have a finite lifetime and, therefore, a vanishing coverage in the thermodynamic limit. Endemic realizations, on the other hand, have an infinite lifetime and their coverage is equal to 1. Below the epidemic threshold, all realizations are trivially finite. Above the threshold, there is a non-null probability $P_{\text {end }}(\lambda)$ that a realization that starts at a single node becomes endemic, making $P_{\text {end }}$ a good order parameter of the phase transition. Akin to the role of the average size of finite clusters in standard percolation [28], in our approach the role of susceptibility is played by the average lifetime of finite realizations $\bar{T}(\lambda)$, which diverges at $\lambda_{c}$ both from below and from above.

In finite systems, the major problem is to determine when a realization is endemic or not. One possibility is to declare a realization as endemic whenever its coverage reaches 1 . However, from a computational point of view, this option is too costly. We therefore take advantage of the following fact. In an infinite size system, whenever the coverage of a realization reaches a finite fraction (even small), the probability of the realization being endemic is 1 . Then, in finite systems, we declare a realization as endemic whenever its coverage reaches a predefined value (in our case $C=0.5$, see Supplemental Material for tests with other values [25]) and stop the realization at this point. Then, we can measure the average lifetime of finite realizations $\bar{T}(\lambda, N)$ and the position of its 

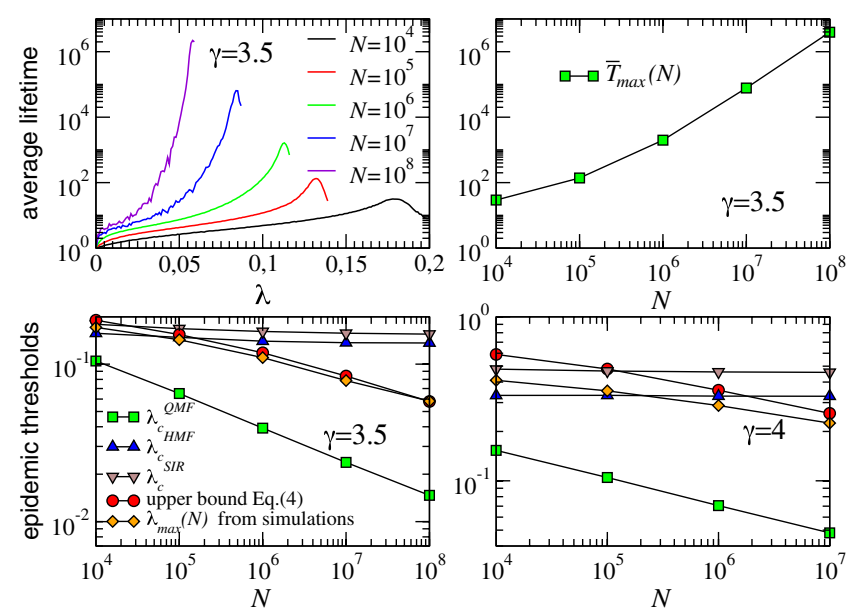

FIG. 1 (color online). Top plots (single network realization): (left) average lifetime of finite realizations for PL random graphs with $\gamma=3.5, k_{\min }=3$, and different sizes; (right) divergence of the height of the peak in $\bar{T}(\lambda)$ as a function of the network size. Bottom plots (averages over 5 network realizations): Epidemic thresholds as measured in numerical simulations in PL networks of different sizes, $\gamma=3.5, k_{\min }=3$ and $\gamma=4, k_{\min }=2$. It also shows the upper bound predicted by our theory as found from a numerical solution of Eq. (4) with $\tau(k, \lambda)$ measured in numerical simulations. For the sake of comparison, we also show the QMF, HMF, and SIR thresholds.

peak, which we take as the estimate of the epidemic threshold for finite systems. Finally, we note that the method can be applied starting from any node of the network with identical results as far as the position of the threshold is concerned. Here, to minimize the fluctuations of $\bar{T}(\lambda, N)$ close to the critical point, we always start our simulations from the node with highest degree.

Figure 1 shows the result of this program in random PL networks generated with the uncorrelated configuration model [29]. The average lifetime $\bar{T}(\lambda, N)$ behaves as an effective susceptibility and, thus, we estimate the epidemic threshold for a finite network as the position of its peak $\lambda_{\max }(N)$. These estimates are shown in the bottom plots and compared with the upper bound given by a numerical solution of Eq. (4) and where $\tau(k, \lambda)$ is obtained from numerical simulations. As it can be clearly seen, the upper bound predicted by our theory is in very good agreement with numerical simulations, even for $\gamma=4$, a network clearly "unclustered" according to [14].

Note that, due to the approximation made in Eq. (2), our theory neglects the propagation of the epidemic mediated only by connected nodes, which is the approach taken in the HMF theory. Therefore, one should expect that the true upper bound for the real epidemic threshold is the minimum between the estimation given by Eq. (4) and $\lambda_{c}^{\mathrm{HMF}}$. From this perspective, it is surprising that the epidemic threshold measured from simulations is higher than $\lambda_{c}^{\mathrm{HMF}}$ for small system sizes. Note, however, that the HMF theory of the SIS dynamics completely neglects dynamical correlations. These correlations account for the fact that, whenever a node is infected, there is a high probability for the node that infected it to still be infected. Therefore, the number of neighbors available to an infected node to further propagate the epidemics is, in most cases, its degree minus 1. Consequently, a better upper bound for the local propagation of the dynamics is given by the HMF theory of the susceptible-infected-recovered (SIR) model; that is, $\lambda_{c}^{\mathrm{SIR}}=\langle k\rangle /\langle k(k-1)\rangle$. Bottom plots of Fig. 1 show the estimation of $\lambda_{c}^{\text {SIR }}$, which is always above the real threshold.

In summary, the behavior of the SIS epidemic threshold in networks depends on a delicate balance between their local and global properties. Both HMF and QMF theories are constructed by considering only the local dynamics of infections among nearest neighbors, and thus fail to provide a correct description. Here we have presented a theoretical approach to epidemics in networks, built upon previously sketched concepts, that takes into account the full network structure, and that considers reinfection events among nodes not directly connected, i.e., mediated by chains of other nodes. Our theoretical analysis, while based in some (reasonable) approximations, is well backed up by means of reliable numerical evidence. The main conclusion of both approaches is that the epidemic threshold in the SIS model is effectively null in the thermodynamic limit in all random small-world networks with a degree distribution decaying slower than exponentially. Beyond this remarkable result, our work highlights the subtle role that dynamical correlations might play in nonequilibrium heterogeneous systems near criticality.

M. B. acknowledges financial support from the Spanish MICINN Project No. FIS2010-21781-C02-02, Generalitat de Catalunya Grant No. 2009SGR838, and by the ICREA Academia prize, funded by the Generalitat de Catalunya. R. P.-S. acknowledges financial support from the Spanish MICINN, under Project No. FIS2010-21781-C02-01 and additional support through ICREA Academia, funded by the Generalitat de Catalunya.

[1] R. Albert and A.-L. Barabási, Rev. Mod. Phys. 74, 47 (2002).

[2] S. N. Dorogovtsev and J.F.F. Mendes, Evolution of Networks: From Biological Nets to the Internet and $W W W$ (Oxford University Press, Oxford, England, 2003).

[3] M.E. J. Newman, Networks: An Introduction (Oxford University Press, Oxford, England, 2010).

[4] R. Pastor-Satorras and A. Vespignani, Phys. Rev. E 65, 036104 (2002).

[5] R. Cohen, S. Havlin, and D. ben-Avraham, Phys. Rev. Lett. 91, 247901 (2003).

[6] W. Goffman and V. A. Newill, Nature (London) 204, 225 (1964).

[7] J. Leskovec, L. A. Adamic, and B. A. Huberman, ACM Trans. Web 1, 5 (2007). 
[8] R. M. Anderson and R. M. May, Infectious Diseases in Humans (Oxford University Press, Oxford, England, 1992).

[9] J. Marro and R. Dickman, Nonequilibrium Phase Transitions in Lattice Models (Cambridge University Press, Cambridge, England, 1999).

[10] R. Pastor-Satorras and A. Vespignani, Phys. Rev. Lett. 86, 3200 (2001).

[11] C. Castellano and R. Pastor-Satorras, Phys. Rev. Lett. 105, 218701 (2010).

[12] C. Castellano and R. Pastor-Satorras, Sci. Rep. 2, 371 (2012).

[13] A. V. Goltsev, S. N. Dorogovtsev, J. G. Oliveira, and J. F. F. Mendes, Phys. Rev. Lett. 109, 128702 (2012).

[14] H. K. Lee, P.-S. Shim, and J.D. Noh, Phys. Rev. E 87, 062812 (2013).

[15] S. N. Dorogovtsev, A. V. Goltsev, and J. F. F. Mendes, Rev. Mod. Phys. 80, 1275 (2008).

[16] A. Barrat, M. Barthélemy, and A. Vespignani, Dynamical Processes on Complex Networks (Cambridge University Press, Cambridge, England, 2008).

[17] M. Boguñá, C. Castellano, and R. Pastor-Satorras, Phys. Rev. E 79, 036110 (2009).
[18] S. C. Ferreira, C. Castellano, and R. Pastor-Satorras, Phys. Rev. E 86, 041125 (2012).

[19] D. Chakrabarti, Y. Wang, C. Wang, J. Leskovec, and C. Faloutsos, ACM Trans. Inf. Syst. Secur. 10, 1 (2008).

[20] P. Van Mieghem, J. Omic, and R. Kooij, IEEE ACM Trans. Netw. 17, 1 (2009).

[21] S. Gómez, A. Arenas, J. Borge-Holthoefer, S. Meloni, and Y. Moreno, Europhys. Lett. 89, 38009 (2010).

[22] F. Chung, L. Lu, and V. Vu, Proc. Natl. Acad. Sci. U.S.A. 100, 6313 (2003).

[23] T. Vojta, J. Phys. A 39, R143 (2006).

[24] S. Chatterjee and R. Durrett, Ann. Probab. 37, 2332 (2009).

[25] See Supplemental Material at http://link.aps.org/ supplemental/10.1103/PhysRevLett.111.068701 for additional details, numerical results, and theoretical methods.

[26] J. A. Hołyst, J. Sienkiewicz, A. Fronczak, P. Fronczak, and K. Suchecki, Phys. Rev. E 72, 026108 (2005).

[27] H. D. Rozenfeld, S. Havlin, and D. ben Avraham, New J. Phys. 9, 175 (2007).

[28] D. Stauffer and A. Aharony, Introduction to Percolation Theory (Taylor \& Francis, London, 1994), 2nd ed.

[29] M. Catanzaro, M. Boguñá, and R. Pastor-Satorras, Phys. Rev. E 71, 027103 (2005). 